\title{
The independence of endogenous and exogenous temporal attention
}

\author{
C. R. McCormick ${ }^{1}$ - R. S. Redden - M. A. Lawrence - R. M. Klein
}

Published online: 31 July 2018

(C) The Psychonomic Society, Inc. 2018

\begin{abstract}
Temporal attention is the focusing of perceptual resources at a particular point in time. Valid temporal cue information has the capability to improve performance by reducing reaction times, while invalid information has the possibility of impairing performance. The performance difference between valid and invalid conditions is called a temporal cueing effect (TCE). We explored how different alerting mechanisms interact with a participant's ability to utilize temporal information cues, using the Kingstone (The Quarterly Journal of Experimental Psychology, 44(1), 69-104, 1992) temporal cueing paradigm. Extracting the alerting procedure from Lawrence and Klein (Journal of Experimental Psychology: General, 142(2), 560-572, 2013), one of two different temporally contingent warning signals were presented to participants during a trial. The "hi-intensity" warning signal increases intensity and elicits both exogenous and endogenous alerting mechanisms. The "no-intensity" warning signal is isointense relative to baseline and elicits only endogenous alerting mechanisms. Two experiments conducted previously using a discrimination task showed interference between the signal intensity and task difficulty, where the "no-intensity" signal failed to elicit TCEs. In the present study, we implemented a detection task, reducing the mental effort required for a response. The results showed equal TCEs in both signal conditions. We argue for independence of these alerting mechanisms, by way of Sternberg's (Acta Psychologica, 30, 276-315, 1969) additive factor method. Arguments contrasting what mechanism is being impacted by this paradigm are further outlined.
\end{abstract}

Keywords Temporal attention $\cdot$ Temporal cueing effects $\cdot$ Endogenous alerting $\cdot$ Exogenous alerting

Klein and Lawrence $(2011,2012)$ developed a taxonomy of attention in which resource allocation in the domains of space, time, and activity can be controlled exogenously or endogenously. One method for exploring the endogenous control of attention in time is the temporal cueing paradigm pioneered by Kingstone (1992). This is a simple, novel, and fruitful method used to explore the control of temporal attention, whereby alphabetic cues are employed to inform participants of various experimental contingencies so that their attention could be modified appropriately. Kingstone modeled this methodology on the Posner cueing paradigm (Posner, Snyder, \& Davidson, 1980) for exploring endogenously generated shifts of visuospatial attention. In his fourth experiment, Kingstone had observers engage in a two-alternative speeded-choice task in which targets for response were preceded by alphabetic

C. R. McCormick

colin.mccormick@dal.ca

1 Dalhousie University, Halifax, NS, Canada temporal cues that indicated the likely time of target onset; after either a short or long stimulus-onset asynchrony (SOA). The cues provided accurate temporal information $80 \%$ of the time. For targets presented at the early SOA, Kingstone found a reaction time (RT) advantage following valid (relative to invalid) temporal cues, an effect that has been dubbed a temporal cueing effect (TCE). Within this paradigm, data from the shorter SOA provide the temporal cueing effect of interest because, as noted by Kingstone (this is a paraphrase), "performance at the long SOA is highly contaminated by the fact that subjects know by default that if a target stimulus does not appear at the short SOA, then it must appear at the long one" (1992, pp. 93-94). Thus, when the target is expected at the short SOA but is "invalidly" presented at the long SOA, participants can adjust or "reorient" their temporal expectancy once the short SOA elapses without presentation of the target.

It is important to highlight Kingstone's methodological and empirical contributions to the field of temporal attention through his invention of this paradigm, that we will refer to as "Kingstonian," because his contributions are often ignored. 
For example, in their highly cited neuroimaging study of spatial and temporal expectancies, Coull and Nobre (1998) minify Kingstone's paradigmatic contribution, saying in their introduction, "By contrast, there has been little or no research investigating attentional orienting to a particular point in time (but see Kingstone, 1992)" (p. 7426). After presenting their interesting findings, they say,

Although the benefits of spatial cueing are well established, this is the first demonstration that such benefits also can manifest themselves when attention is paid to moments in time. One exception is the study by Kingstone (1992), which investigated the interaction between attending to form and attending to temporal interval but did not investigate temporal cueing per se. (p. 7431)

Also, in a recent paper discussing the relation between temporal attention (as explored using the temporal cueing paradigm developed by Kingstone) and alerting (as explored in warning signal experiments), Weinbach and Henik, (2012) fail to cite Kingstone (1992), opting instead to cite Coull and Nobre (1998) and a review paper by Correa (2010). The Correa paper, which was published in Nobre and Coull's (2010) book Attention and Time, also neglects to cite Kingstone. ${ }^{1}$

Whereas endogenous control is top down and volitional, exogenous control is bottom up and reflexive (Jonides, 1981). This is an issue within the current literature, as pointed out by Weinbach and Henik (2012, 2013), as these are entangled in the methodological design of many temporal cueing paradigms. In accord with Klein and Lawrence's (2012) emphasis on the applicability of endogenous and exogenous modes of control in the domain of time, Lawrence and Klein (2013) developed a methodology suitable for isolating endogenous versus exogenous control of alerting, or attention in time. Alerting is defined as the preparation and maintenance of arousal to process stimuli (Posner \& Petersen, 1990). In Lawrence and Klein's (2013) design, participants were presented with continuous mono (i.e., diotic) white noise. Signals were presented by briefly shifting from mono to stereo (i.e., dichotic) sound (uncorrelated white noise to both ears). Even without a global increase in amplitude, this change is easily detected by observers. Lawrence and Klein used these signals with or without a global increase in intensity (i.e., isointense). ${ }^{2}$

\footnotetext{
${ }^{1}$ Whereas we want to give credit to Kingstone for his seminal contribution in extending Posner's endogenous spatial cueing paradigm to the temporal domain, in agreement with the words of an anonymous reviewer, we certainly do not mean to dismiss "the contribution of Nobre and Coull to the temporal literature, which (regardless of whether they were first) is undeniable, and surely had a great impact on the field in numerous follow-up studies."

${ }^{2}$ The no-intensity change condition is analogous to an isoluminant color change in vision.
}

A signal that does not increase in intensity allows us to explore purely endogenous control systems of temporal attention, as there is no attention-grabbing component; that is, signals with no intensity change require a more volitional form of processing. The individual is alerted in a volitional manner, as they can use top-down processing to increase arousal to better process stimuli. Signals that do increase in intensity reflexively grab the attention of an individual and, when they can be used to predict the time of the target, incorporate both exogenous and endogenous control systems. In the present study, we will be using this signal intensity manipulation, in which a change from mono to stereo presentation of noise is or is not accompanied by an intensity change.

Implementing stereo signals with and without an intensity change into a Kingstonian temporal cueing paradigm allows us to compare purely endogenous temporal attention to a condition in which both endogenous and exogenous temporal attention are involved, allowing us to explore the interaction between these two modes of temporal attention. Signals that do not increase in intensity will be referred to as no-intensity signals, while signals that do increase in intensity will be referred to as hi-intensity signals. In addition, combining these methodological features will allow us to ameliorate an inherent limitation in the prototypical Kingstone temporal cueing paradigm: The cue indicates the likely fore-period information and simultaneously notifies the observer to start their internal timer. This means the endogenously generated preparation generated by the meaning (i.e., temporal informativeness) of the cue from the exogenously generated arousal rendered from the onset of the cue are problematically paired together (Weinbach \& Henik, 2012). Although alerting and temporal cueing have been identified as different mechanisms, their effects are typically confounded (Weinbach \& Henik, 2013). We hope to alleviate this with our method, which allows for the separation of these two distinct, but complementary behavioral requirements of a temporal cueing paradigm: interpreting the relevant temporal information, and starting the appropriate internal timer. The informative cue akin to that used by Kingstone will be presented on screen to provide temporal information, and an auditory signal (as in Lawrence \& Klein, 2013) will subsequently indicate the requisite instruction to start the timer set according to the information provided by the cue.

We (McCormick, Redden, Lawrence, \& Klein, 2017) conducted two prior experiments implementing these features in a two-alternative forced-choice discrimination task (see Fig. 1). In one experiment, the no-intensity and hi-intensity signals were randomly intermixed in the same block of trials. We found a main effect of signal intensity but no TCE. This failure led to the hypothesis that the amount of mental effort required to detect the no-intensity warning signal may have interfered with an observer's ability to effectively make use of the temporal cue. When the signal types are mixed, one must always 


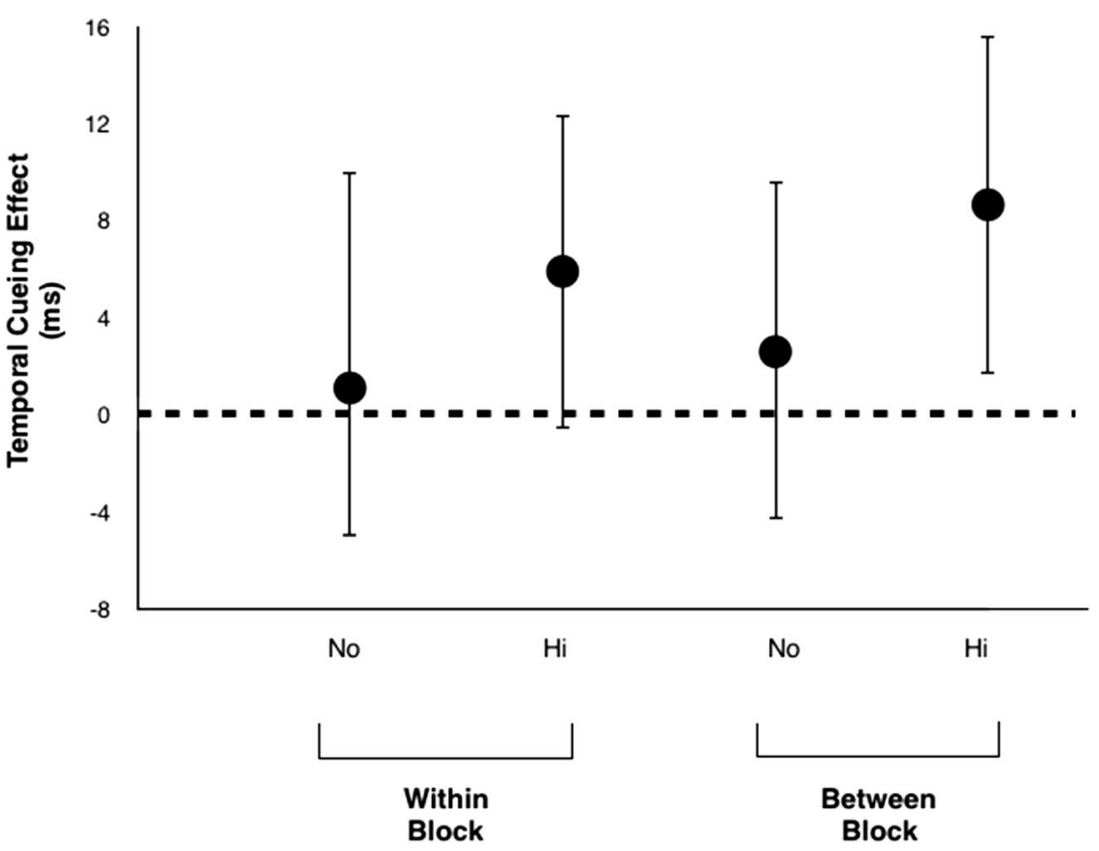

Fig. 1 Left half: A within-block intensity discrimination paradigm looking at the temporal cueing effects (invalid RT - valid RT). This manipulation $(n=40)$ produced no temporal cueing effects (TCEs) in either the no-intensity or the hi-intensity condition. Right half: A

between-block intensity discrimination paradigm looking at temporal cueing effects across two signal intensities. This manipulation $(n=40)$ produced a TCE in the hi-intensity condition, but not in the no-intensity condition. Error bars are 95\% confidence intervals

be in a mental state to anticipate a no-intensity warning signal, lest one miss the signal. Following a similar logic in a different domain (Shore, McLaughlin, \& Klein, 2001), we tested this hypothesis in the second experiment by presenting the two intensity conditions in separate blocks. The main effect of intensity was replicated, with faster responses with a hiintensity signal compared with the no-intensity signal. Furthermore, in support of our mental effort hypothesis, we obtained a TCE with the hi-intensity signals and not with the no-intensity signals. In these two preliminary studies, then, we saw no TCE when signal intensities were mixed, nor when using exclusively no-intensity signals in combination with the temporal cueing paradigm. Accordingly, we were left without any clear conclusions regarding how the two modes of alerting may interact.

The hypothesis that mental effort may mitigate TCEs has precedent in the literature (Correa, Lupiáñez, Milliken, \& Tudela, 2004). Correa et al. showed that TCEs can be modulated by stimulus-response demands, whereby robust TCEs were obtained in detection tasks but were considerably less robust when the task was discrimination. Additionally, occupying working memory during a temporal cueing experiment can eliminate a TCE (de la Rosa, Sanabria, Capizzi, \& Correa, 2012). This support, in combination with our pilot findings, led us to simplify our target task from discrimination to detection. Based on our earlier robust finding of an effect of signal intensity (exogenous temporal attention) and our expectation (based on Correa et al., 2004) that when using a detection task we would obtain a TCE (endogenous temporal attention), our key question is whether these two forms of control of temporal attention would be additive or interactive.

\section{Method}

\section{Participants}

Fifty participants were run in this experiment in a computer laboratory. Data collection was conducted in groups of four to 10 , until 40 participants (mean age $=20.98$ years, 11 males, seven left-handed) met our trial count criterion (see Results section). Previous studies measuring a behavioral effect have tested an average of $n=9.5(\min =7, \max =12)^{3}$ subjects per condition. To ensure that our methods would be at least as powerful as the studies existing in the literature, we elected to aim for a sample that quadrupled these previous sample sizes. A power calculation based on a cueing effect ( $8 \mathrm{~ms} ; d$ $=.49$ ) in one condition from our pilot studies reported in the Introduction (McCormick et al., 2017) showed that with a sample size of 40 and a Type I error of .05, the a priori power of the current experiment was 0.85 . All participants were undergraduate students at Dalhousie University. Participants had

\footnotetext{
${ }^{3}$ Correa et al. (2004) Experiment 1: $N=8$ per condition, Experiment 2a: $N=8$ per condition, Experiment 2b: $N=7$ per condition; Kingstone (1992) Experiment 4: $N=10$; Correa, Lupiáñez, Madrid, and Tudela (2006): $N=$ 12; Coull and Nobre (1998): $N=12$.
} 
normal or corrected-to-normal vision and did not have any known neurological or cognitive disorders.

\section{Apparatus}

The experiment was presented on ten 24-inch Mac monitors connected to a Mac Mini running OS X with a $2.66 \mathrm{GHz}$ Intel Core 2 Duo processor and a NIVIDIA GeForce $9400256 \mathrm{MB}$ graphics card. A set of 10 headphone monitors (Sony MDR101LP) were used. Acceptable volume settings ranged between three and five. Participants sat at a maximum distance of $102 \mathrm{~cm}$ from the screen. Game-pad controllers [Xbox 360 wired controllers] were used by participants to make responses. ${ }^{4}$

\section{Stimuli}

Temporal cues, which could take the form of either the letter "S" or "L" (.5 degrees of visual angle; DVA) with equal probability were presented at the center of the screen until the presentation of the target. These cues were blue (RGB: $0,0,255)$ on a gray (RGB: 119, 119, 199) background. Targets took the form of circles (.5 DVA) that appeared at the center of the screen. Targets were either black (RGB: 0, 0,0 ) or white (RGB: $255,255,255$ ). Participants received onscreen feedback in the form of a single digit (.5 DVA) after making their response, which reflected their reaction time on that trial to the 10th of a second.

Mono white noise was constantly presented throughout a trial. A sampling frequency of $44100 \mathrm{~Hz}$ was used. Auditory warning signals were presented in the form of stereo white noise for a duration of $100 \mathrm{~ms}$. In the 'no-intensity warning signal condition, there was no change in intensity relative to the mono baseline. In the hi-intensity warning signal condition, the ambient volume was doubled.

\section{Procedure}

Participants were instructed that the temporal information provided by cues would help optimize performance as the cues indicated with high probability $(80 \%)$ whether the fore period between the auditory warning signal and the target would be short or long. A practice block was conducted consisting of 40 trials that were not included in the analysis.

Mono noise was presented in both ears continuously throughout the task. Trials began with a blue letter in the center of a gray screen. This letter was a temporal information

\footnotetext{
${ }^{4}$ Gamepad controllers were used in the place of the traditional keyboard response. This allows for the filtering of response data, as we can determine when participants are making specific inappropriate responses (single trigger presses, pressing the trigger multiple times, etc.).
}

cue that could either be an $\mathrm{S}$ or an $\mathrm{L}$ to represent a probable $400 \mathrm{~ms}$ or $1,600 \mathrm{~ms}$ fore period between the later auditory warning signal and the presentation of the target, respectively. These cues were $80 \%$ predictive. The cue remained on-screen until the target was presented. The auditory warning signal was presented after a random exponential (i.e., "nonaging") interval within the range of 2 to 10 seconds (mean $=4 \mathrm{~s}$ ) to indicate the target was imminent. The auditory warning signal was either the hi-intensity or no-intensity change from mono to stereo noise and was presented for a duration of $100 \mathrm{~ms}$. Either 400 or 1,600 ms after this auditory cue, a black or white target was presented for either $1,000 \mathrm{~ms}$ or until the participant responded. Simple detection responses were made by pressing both triggers on the gamepad. Participants were instructed that they were required to press the response apparatus at least halfway down to register a response. If participants responded before the target was on the screen, then a message that read "Too Early!" was presented in red (RGB: 255, 0, 0). If they failed to respond during this $1,000 \mathrm{~ms}$ window, then a message that read "Miss!" was presented in red. This experiment has 40 trials per block, with 12 blocks in total (Fig. 2).

\section{Results}

\section{Data preprocessing}

Before beginning this experiment, we decided to only include participants with greater than $75 \%$ of trials completed. The was consistent with our two prior pilot experiments (see Fig. 1). We first removed any trials that we called anticipatory responses, which included responses after a signal but before a target (12.9\%), or responses faster than $150 \mathrm{~ms}(2.2 \%)$. We then eliminated trials that were slower than $600 \mathrm{~ms}(1.1 \%)$, and when participants missed responding to the target $(.25 \%)$. For the remainder of trials, we eliminated trials based on inappropriate trigger responses, which included no maximum response, pressing only one trigger, and multiple responses $(6.05 \%)$. After excluding these trials, we checked to see who met our previously set criterion for percentage of trials completed, which resulted in the removal 10 of the 50 participants.

\section{Statistical tests}

Results from 40 participants (11 male, seven left-handed, mean age $=20.98$ years) were included in statistical analysis . As noted in the Introduction, once the short SOA has expired without a target, performance on targets presented at the long SOA is "confounded" by the possibility that temporal reorienting will obviate the cueing manipulation. Therefore, our primary analyses will focus on response times to targets presented at the short SOA. 

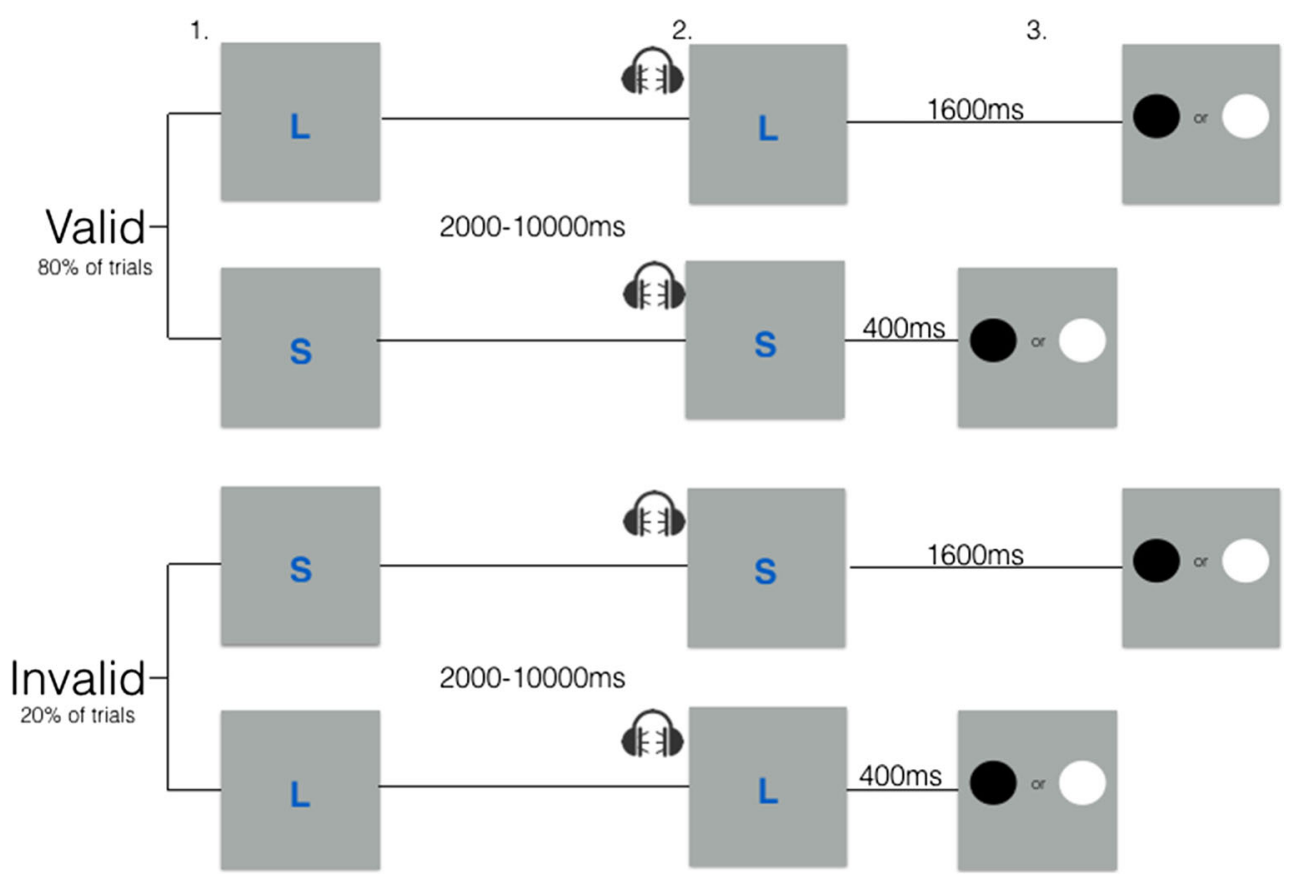

Fig. 2 Four different trial runs in the current experiment: valid long, valid short, invalid short, and invalid long. White noise is played throughout the task. 1. The trial begins with either a short (S) or long (L) temporal cue. This indicates (with $80 \%$ probability) whether the target will follow the signal (Panel 2) by a short or long SOA. 2. After a random fixation interval $(2-10 \mathrm{~s})$, an auditory signal is presented through headphones. This signal is a 100 -ms change from mono to stereo noise. Importantly,

We performed a 2 (signal intensity) $\times 2$ (cue condition) ANOVA on mean RT to targets presented at the short $\mathrm{SOA}^{5}$ using the EZ package within the R statistics software (Lawrence, 2016; R Core Team, 2016). We observed a significant effect for the cue condition, $F(1,39)=37.76, p<.001$, $\eta_{\mathrm{p}}{ }^{2}=.068$, with faster RTs for the valid (284 ms) than for the invalid (299 ms) cue condition. There was also a significant effect of signal intensity, $F(1,39)=32.23, p<.001, \eta_{\mathrm{p}}{ }^{2}=.08$, with faster RTs for the hi-intensity condition $(278 \mathrm{~ms})$ than for the no-intensity condition ( $295 \mathrm{~ms})$. There was no interaction effect between cue condition and signal intensity, $F(1,39)=$ $.051, p=.823, \eta_{\mathrm{p}}^{2}<.001$.

The additivity seen here in reaction time suggests that the two forms of temporal attention are independent and operating at different stages of processing. If this is really the case, then according to Sternberg (1969), we should also see an additive

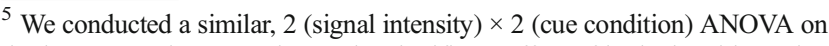
the long SOA data. We observed a significant effect of both signal intensity, $F(1,39)=13.97, p<.001, \eta_{\mathrm{p}}{ }^{2}=.012$, and cue condition, $F(1,39)=19.26, p<$ $.001, \eta_{\mathrm{p}}{ }^{2}=.024$. In comparison to our short SOA analysis, we observed faster RTs in the invalid condition (294 ms) compared with the valid condition (305 $\mathrm{ms})$. This is the pattern that would be expected if the dynamic reorienting that can happen after the short SOA elapses without the expected target is more effective than the static expectation that was put into play when the temporal cue was delivered seconds earlier.
}

this signal either increased in intensity from the background stereo noise (hi-intensity signal) or maintained the same intensity (no-intensity signal) as the background. 3. After the short or long SOA, a white or black target $(50 / 50)$ is presented at fixation. Participants are instructed to press both triggers as soon as they detect a circle. If participants respond within the allotted $1,000 \mathrm{~ms}$, then feedback of their reaction time speed is presented at the center of the screen (e.g., 234)

pattern if we analyze the variances of RT. We therefore performed a 2 (signal intensity) $\times 2$ (cue condition) ANOVA on RT variance. In this analysis we observed significant main effects of cue condition, $F(1,39)=14.78, p<.001, \eta_{\mathrm{p}}{ }^{2}=$ .05 , and signal intensity, $F(1,39)=11.37, p=.0017, \eta_{\mathrm{p}}^{2}=$ .048 . The interaction between cue condition and signal intensity, $F(1,39)=2.32, p=.136, \eta_{\mathrm{p}}{ }^{2}=.008$, was not significant (Fig. 3). ${ }^{6}$

\section{Discussion}

Our analysis demonstrates that the two forms of temporal attention explored here are independent: temporal cueing (endogenous temporal attention) and signal intensity (exogenous temporal attention) had additive effect on both RT and its variance. Following Sternberg's (1969) additive factors method, this pattern strongly implies that these two factors and therefore these two forms of temporal attention are affecting different stages of processing,

\footnotetext{
${ }^{6}$ We additionally performed an analysis that included all 50 participants and found the same pattern of results in both the reaction time analysis and the Sternberg analysis, but with a greater amount of variance.
} 


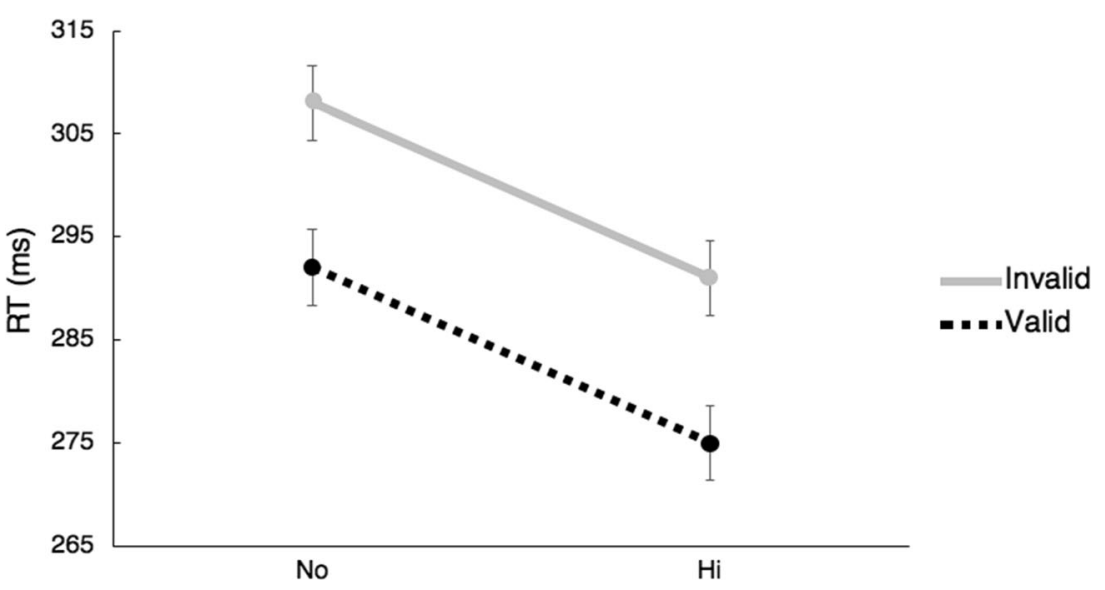

Fig. 3 A within-block, mixed-intensity detection paradigm run in the current experiment comparing the mean reaction times of valid (black dashed line) and invalid (gray solid line) cues across our two signal intensities (error bars are Fisher's least significant difference)

Whereas we can be confident that "different" stages of processing are affected by the two forms of temporal attention explored here, from the results of the present experiment we cannot make confident assertions about which stages of processing each form is affecting. That noted, there are some hints in the literature. Posner (Posner \& Boies, 1971; Posner, Klein, Summers, \& Buggie, 1973) generated converging evidence for his proposal (Posner, 1975) that the alertness or preparation generated in warning signal studies did not affect the rate of accumulation of information about the subsequent target, but instead affected when the attention/decision system would consult this accumulating information. In particular, the observed responds sooner when alert than when not alert, which predicts that so long as targets are not brief or masked, then the reaction time advantage associated with alertness will be accompanied by a disadvantage in accuracy (a prediction confirmed by Posner et al., 1973). Lawrence and Klein (2013) pointed out that in the kinds of studies on which Posner's proposal was based, the warning signals could be endogenously used to focus attention in time but also entailed the possibility of exogenous temporal attention (arousal) because the warning signals entailed a change on intensity. Importantly, Lawrence and Klein confirmed Posner's proposal while also demonstrating that the speed-accuracy trade-off it predicted was confined to high-intensity signals that could be used to prepare. Thus both Posner's proposal and Lawrence and Klein's findings strongly suggest that the effect of highintensity signal is probably not affecting the rate of information accrual about the target and instead is affecting when the attention/decision to initiate a response is made. Although one might have imagined that with a simple RT task, like we have used here, the temporal cueing manipulation might be affecting such a late stage, the additivity we have found provides evidence against this. Moreover, there are several studies using the Kingstonian paradigm (Correa, Lupiáñez, Madrid, \& Tudela, 2006; Correa, Sanabria, Spence, Tudela, \& Lupiáñez, 2006) that suggest that early stages of processing are enhanced by temporal expectancy. These findings from the literature suggest that the temporal cueing paradigm, that we believe is primarily manipulating temporal attention endogenously, is operating on an early stage of processing and that the effect of a hi-intensity signal, that we believe has its effect primarily exogenously, is affecting a later (decision or response) stage of processing. We offer this simply as our best guess at this time. Further experimentation is needed to be confident.

Future experiments should continue with this tradition of measuring independence of alerting and preparation stages within the different domains of the Klein and Lawrence (2012) attention model. The isolation of the temporal cue and alerting signal can provide the ability to study their effects independently, something that has been missing from the current body of literature (Weinbach \& Henik, 2012). One possible next step is to isolate which stage of processing was impacted by these manipulations to further expand our knowledge related to the independence of these alerting mechanisms. Developing a paradigm with a discrimination task in which we can successfully observe TCEs with both the hi-intensity and no-intensity signals would be ideal, as this would allow us to observe the relation between speed and accuracy under these two signaling conditions. It is important to continue to develop this taxonomy of attention and explore how it might incorporate novel forms of temporal attention (such as that elicited when we experience rhythmic stimuli; Barnes \& Jones, 2000; for a review, see Nobre \& Ede, 2018) because we believe that the distinction between endogenous and exogenous control is both important and too often ignored. 


\section{References}

Barnes, R., \& Jones, M. R. (2000). Expectancy, attention, and time. Cognitive Psychology, 41(3), 254-311.

Correa, Á. (2010). "Enhancing behavioural performance by visual temporal orienting." in A. C. Nobre \& J. T. Coull (Eds.), Attention and time (pp. 357-370). Oxford, UK: Oxford University Press.

Correa, Á., Lupiáñez, J., Madrid, E., \& Tudela, P. (2006). Temporal attention enhances early visual processing: A review and new evidence from event-related potentials. Brain Research, 1076(1), 116128

Correa, Á., Lupiáñez, J., Milliken, B., \& Tudela, P. (2004). Endogenous temporal orienting of attention in detection and discrimination tasks. Attention, Perception, \& Psychophysics, 66(2), 264-278.

Correa, Á., Sanabria, D., Spence, C., Tudela, P., \& Lupiáñez, J. (2006). Selective temporal attention enhances the temporal resolution of visual perception: Evidence from a temporal order judgment task. Brain Research, 1070(1), 202-205.

Coull, J. T., \& Nobre, A. C. (1998). Where and when to pay attention: The neural systems for directing attention to spatial locations and to time intervals as revealed by both PET and fMRI. Journal of Neuroscience, 18(18), 7426-7435.

de la Rosa, M. D., Sanabria, D., Capizzi, M., \& Correa, A. (2012). Temporal preparation driven by rhythms is resistant to working memory interference. Frontiers in Psychology, 3. https://doi.org/ 10.3389/fpsyg.2012.00308

Jonides, J. (1981). Voluntary versus automatic control over the mind's eye. Attention and Performance, 9, 187-203.

Kingstone, A. (1992). Combining expectancies. The Quarterly Journal of Experimental Psychology, 44(1), 69-104.

Klein, R. M., \& Lawrence, M. A. (2011). The modes and domains of attention. In M. I. Posner (Ed.), Cognitive neuroscience of attention (2nd ed., pp. 11-28). New York, NY: Guilford Press.

Klein, R. M., \& Lawrence, M. A. (2012, May). Pursuing a productive taxonomy of attention. Paper presented at On the Future of Attention Research and Practice: Defining, Measuring and Repairing the Networks of Attention: An International Symposium, Halifax, Canada.
Lawrence, M. A. (2016). EZ: Easy analysis and visualization of factorial experiments (R Package Version 4.4-0) [Computer software]. Retrieved from https://CRAN.R-project.org/package=ez

Lawrence, M. A., \& Klein, R. M. (2013). Isolating exogenous and endogenous mechanisms of temporal attention. Journal of Experimental Psychology: General, 142(2), 560-572.

McCormick, C. R., Redden, R., Lawrence, M., \& Klein, R. M. (2017, June). On the time-course of cued temporal attention. Poster session at the meeting of the Canadian Society of Brain, Behaviour, and Cognitive Sciences, Regina, Saskatchewan, Canada.

Nobre, K., \& Coull J. T. (2010). Attention and time. New York, NY: Oxford University Press.

Nobre, A. C., \& van Ede, F. (2018). Anticipated moments: temporal structure in attention. Nature Reviews Neuroscience, 19(1), 34.

Posner, M. I. (1975). Psychobiology of attention. In M. S. Gazzaniga \& C. Blakemore (Eds.), Handbook of psychobiology (pp. 441-480). New York, NY: Academic Press. https://doi.org/10.1016/B978-012-278656-3.X5001-7

Posner, M. I., \& Boies, S. J. (1971). Components of attention. Psychological Review, 78(5), 391-408.

Posner, M. I., Klein, R., Summers, J., \& Buggie, S. (1973). On the selection of signals. Memory \& Cognition, 1(1), 2-12.

Posner, M. I., \& Petersen, S. E. (1990). The attention system of the human brain. Annual Review of Neuroscience, 13(1), 25-42.

Posner, M. I., Snyder, C. R., \& Davidson, B. J. (1980). Attention and the detection of signals. Journal of Experimental Psychology: General, $109(2), 160$.

R Core Team. (2016). R: A language and environment for statistical computing. Vienna, Austria: R Foundation for Statistical Computing. Retrieved from https://www.R-project.org/

Shore, D. I., McLaughlin, E. N, \& Klein, R. M. (2001). Modulation of the attentional blink by differential resource allocation. Canadian Journal of Experimental Psychology, 55, 318-324.

Sternberg, S. (1969). The discovery of processing stages: Extensions of Donders' method. Acta Psychologica, 30, 276-315.

Weinbach, N., \& Henik, A. (2012). Temporal orienting and alerting-The same or different?. Frontiers in Psychology, 3, 236.

Weinbach, N., \& Henik, A. (2013). The interaction between alerting and executive control: Dissociating phasic arousal and temporal expectancy. Attention, Perception, \& Psychophysics, 75(7), 1374-1381. 